\title{
ANÁLISIS DE RESPUESTAS LOCALES ANTE FENÓMENOS NATURALES AMENAZANTES Y DINÁMICAS DE CONSTRUCCIÓN DE ESCENARIOS DE RIESGO EN COLOMBIA
}

\section{ANALYSIS OF LOCAL RESPONSES TO NATURAL THREATENING PHENOMENA AND DYNAMICS OF CONSTRUCTION RISK SCENARIOS IN COLOMBIA}

\begin{abstract}
Yolanda Teresa Hernández Peña ${ }^{1}$, Germán Vargas Cuervo ${ }^{2}$
${ }^{1}$ Doctora en Geografía. Profesora Universidad Distrital Francisco José de Caldas, Facultad de Medio Ambiente y Recursos Naturales. Av. Circunvalar Venado de Oro, Bogotá, Colombia, e-mail: ythernandezp@udistrital.edu.co; ${ }^{2}$ Doctor en Ciencias de la Tierra. Profesor Universidad Nacional de Colombia. Carrera 45 No 26-85 Bogotá, Colombia, e-mail: gvargasc@unal. edu.co
\end{abstract}

Rev. U.D.C.A Act. \& Div. Cient. 17(2): 529-539, Julio-Diciembre, 2014

\section{RESUMEN}

Colombia, por su contexto geográfico, geológico, geomorfológico e hidrológico, es un país altamente susceptible a la ocurrencia de fenómenos naturales, como sismos, erupciones volcánicas, inundaciones y movimientos en masa, entre otros. Para mitigar la amenaza y abordar una gestión integral del riesgo, no solamente desde un punto de vista técnico, es necesario tener en cuenta las percepciones e imaginarios de la población expuesta. Este artículo tuvo como objeto contribuir a la gestión del riesgo a partir del análisis y la evaluación de las respuestas locales frente a los fenómenos naturales amenazantes, teniendo como categorías de exploración las percepciones e imaginarios de la población expuesta a riesgo inminente. La investigación, se realizó teniendo como marco la fenomenología y la etnografía y, para ello, se realizaron observaciones de campo, entrevistas, grupos focales y talleres con cartografía de percepción social aplicados para las zonas de influencia del volcán Galeras, volcán del Huila, inundaciones del Canal del Dique y de Tinjacá, sismo probable en Bogotá, deslizamientos en las localidades Ciudad Bolívar y Rafael Uribe, en la ciudad capital. Los resultados muestran diferentes percepciones e imaginarios sobre los fenómenos estudiados, los cuales, no siempre son considerados como amenaza; se encontró la importancia de la memoria histórica sobre los territorios, como un factor estratégico para la prevención de desastres; igualmente, se evidencian los desencuentros de los habitantes de zonas de amenaza respecto a la gestión del riesgo, que ejecutan las autoridades encargadas.
Palabras clave: Imaginarios, percepciones, amenaza, conocimiento local.

\section{SUMMARY}

Colombia by its geographical, geological, geomorphological, hydrological context is highly susceptible to the occurrence of natural phenomena such as earthquakes, volcanic eruptions, floods and landslides among others country. In scenarios of risk, threatening natural phenomena provide a high vulnerability exposed elements. This article aims to contribute to the management of risk from an analysis and evaluation of local responses to threatening natural phenomena as a frame of analysis and imagined perceptions of the population exposed to imminent risk. The research was conducted as a frame phenomenology and ethnography, to do field observations, interviews, focus groups and workshops with social perception mapping applied to the areas of influence of Galeras Vulcano, Huila Vulcano, floods in the Canal del Dique and Tinjacá, probable earthquake in Bogota, landslides in Ciudad Bolívar and Rafael Uribe in Bogota. The results show different perceptions and images about the phenomena studied are not always considered as a threat; the importance of historical memory in the territories as an important strategic element for disaster prevention; also disagreements of people living in hazardous areas regarding risk management made by the responsible authorities are evident.

Keys words: Imaginary, perceptions, hazard, local knowledge. 


\section{INTRODUCCIÓN}

Las personas que habitan en zona de influencia de un fenómeno natural han construido sus propias maneras de entenderlo, a través de la organización de las sensaciones y las percepciones sobre el medio ambiente (Schiffman, 2004). Najmanovich (2008) explica que la percepción no es un proceso mecánico u óptico, es una actividad constructiva, productiva y formativa. Los imaginarios y percepciones, se constituyen en fuente de conocimiento, que se incorpora a los procesos de la vida cotidiana (Berger et al. 2001). Para entender la construcción de escenarios de riesgo y las transformaciones sobre el medio ambiente, Capel (1973), Douglas (1996) y García (2005) coinciden en señalar que el riesgo es producto de una construcción social de los miembros de una determinada sociedad, estableciéndose en un marco de referencia para los individuos.

Geógrafos, como Lindon (2007) y Tuan (2007), han analizado las relaciones perceptuales de los seres humanos con su espacio, que para Tuan (2007), con estas relaciones, se generan entramados simbólicos y de relaciones, que les permite establecer lazos muy fuertes con el territorio. Musset (2009), en estos procesos, involucra relaciones de poder y temas de justicia espacial; también, se relacionan con la manera como son concebidos los territorios y gestionados sus riesgos e inciden en los procesos inacabados de desarrollo (Blakie et al. 1996; Cannon \& Müller-Mahn, 2010; Wisner et al. 2003).

Estos imaginarios y percepciones alimentan las diferentes concepciones sobre la naturaleza, ampliamente estudiadas por Schwarz \& Thompson (1990), Escobar (2011) y Losada (2010); se encuentran presentes en las memorias históricas y en los conocimientos que las poblaciones tienen sobre sus territorios, conformando lo que se ha denominado conocimiento local y popular (Rabey, 1990; Kuclicke, 2007; Mora, 2008) y son componentes fundamentales de las distintas racionalidades: igualitarias, jerárquicas, fatalistas e individualistas en el actuar, frente al medio ambiente y con relación al riesgo (Wildavsky, 1998).

Este artículo, se desarrolla como producto de una investigación doctoral, en el marco del Grupo de Investigación de Geotecnologías de la Universidad Nacional de Colombia, que tiene como objetivo principal realizar un análisis sobre las percepciones e imaginarios que tiene la población expuesta a amenazas y riesgos en momentos críticos o de desastre. Los resultados de este tipo de estudios, se constituyen en insumos para las políticas e intervenciones relacionadas con la gestión del riesgo.

\section{MATERIALES Y MÉTODOS}

En la investigación, se analizaron cuatro escenarios de amenaza y riesgo, así: Primero: escenario de simulacro de terremoto en Bogotá, realizado en octubre de 2009, con una amenaza intermedia de ocurrencia de un sismo de magnitud 4,5 en la escala de Richter y una aceleración máxima probable de $0,24 \mathrm{~g}$ de intensidad, sobre una población cercana a 8 millones de habitantes; Segundo: amenaza de erupción del volcán Galeras, con un escenario de alerta roja para habitantes de la región y desplazamiento a alberges temporales, escenario de contraste volcán del Huila, con la avalancha ocurrida en 1994 y que afectó, principalmente, la comunidad Nasa; Tercero: inundaciones en la costa Caribe, asociadas a la ruptura del Canal del Dique, inundando cerca de $260 \mathrm{~km}^{2}$ de zonas urbanas, cultivos y pastizales para ganadería, afectando unas 30 mil familias, escenarios de contraste Mosquera, Soacha y Tinjacá y Cuarto: deslizamientos activos en Bogotá, localidad de Ciudad Bolívar, afectando continuamente población de bajos recursos, escenario de contraste localidad Rafael Uribe.

En el trabajo de campo, se utilizaron diferentes técnicas etnográficas (Hammersley \& Atkinson, 1994): observación directa, grupos focales para el caso de los deslizamientos en Rafael Uribe y en Ciudad Bolívar, utilizando cartografía de percepción social y entrevistas abiertas, debido a que era necesario indagar sobre imaginarios y percepciones, con los siguientes parámetros: cómo se concibe el fenómeno, si se considera una amenaza, el conocimiento que se tiene sobre sus causas y comportamiento y las relaciones establecidas con éste y con el territorio, para de allí derivar un análisis sobre sus concepciones sobre la naturaleza, percepciones de riesgo, conocimientos locales, sus percepciones sobre sus condiciones de vulnerabilidad, encuentros y desencuentros sobre gestión del riesgo. En el caso del probable sismo en Bogotá, se hicieron preguntas cerradas y abiertas, porque el objetivo fue entrevistar a las personas que participaron en dicho simulacro. Las entrevistas fueron procesadas en el programa Atlas ti. En el cuadro 1, se muestra el proceso metodológico y se señala el momento en que se aplicaron los instrumentos.

Amenazas y riesgos por sismicidad - Bogotá: La capital colombiana se ha zonificado como un área de amenaza sísmica intermedia; se localiza en el altiplano de la Sabana de Bogotá, en La Cordillera Oriental, a una altitud de 2.600 msnm, sobre los 4 grados de latitud Norte y los 74 grados de longitud Oeste. En la zona, se presenta una zona plana a ondulada, donde antes se encontraba una antigua laguna, situado entre los 2.550 y $2.600 \mathrm{msnm}$ y la zona montañosa, que alcanza altitudes de hasta $4.000 \mathrm{msnm}$, conformada por la cadena de cerros, que la rodean. 
Cuadro 1: Esquema general de la investigación.

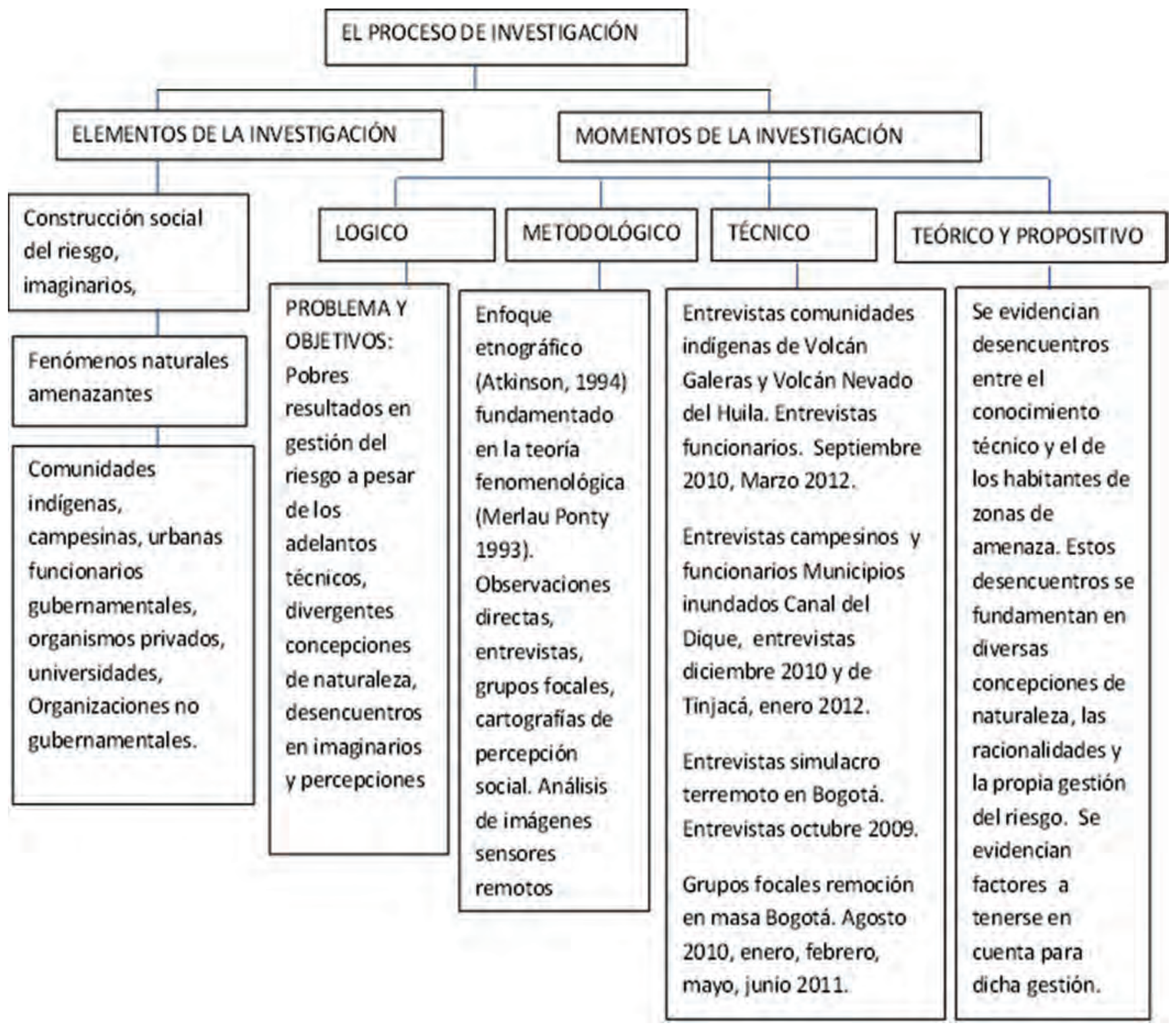

Amenazas y riesgos por erupciones volcánicas - volcán Galeras: El complejo volcánico del Galeras, se ubica en el departamento de Nariño, a $8 \mathrm{~km}$ al occidente de la ciudad de San Juan de Pasto. De acuerdo con el Servicio Geológico Colombiano (2011), el Galeras es un estratovolcán activo, cuyo edificio volcánico tiene una forma cónica, con un cráter despicado en su parte occidental; presenta una altura de 4.276 msnm (Figura 1a).

Según el Servicio Geológico Colombiano (2011), en la zona de influencia, se encuentran siete municipios, entre ellos la ciudad capital -San Juan de Pasto- y una serie de corregimientos y de veredas, que albergan, aproximadamente, 500.000 habitantes. Las zonas de amenaza del volcán Galeras son clasificadas por el Servicio Geológico Colombiano (2011) en alta, media y baja.

La primera, caracterizada por la probabilidad mayor al $20 \%$ que sucedan eventos, como flujos y caídas piroclásticas, flujos de lava y de lodo, ondas de choque y alta concentración de gases, en una máxima distancia de 9,5 km y con una severidad de hasta 5; la segunda, determinada por afectación 


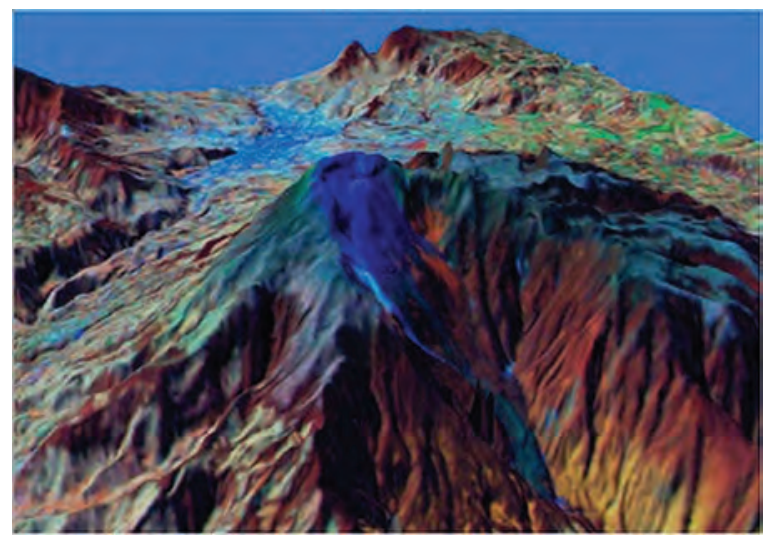

Figura 1a. Cráter volcán Galeras.

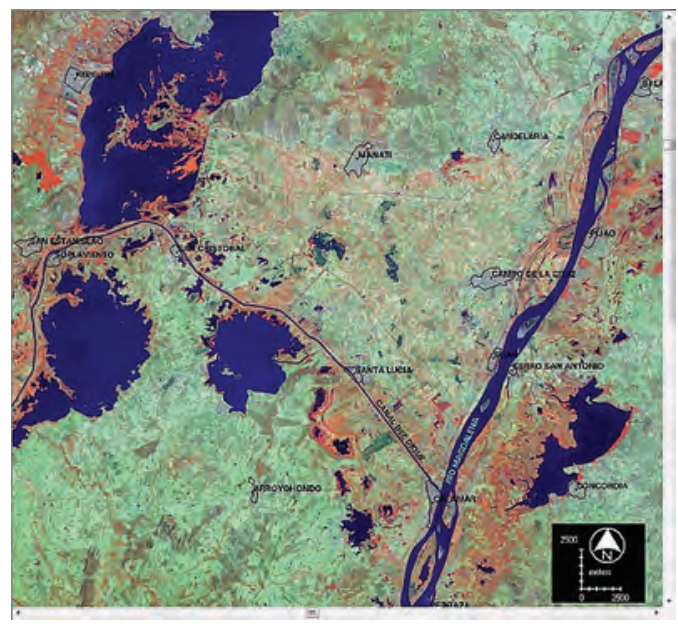

Figura 1b. Canal del Dique.

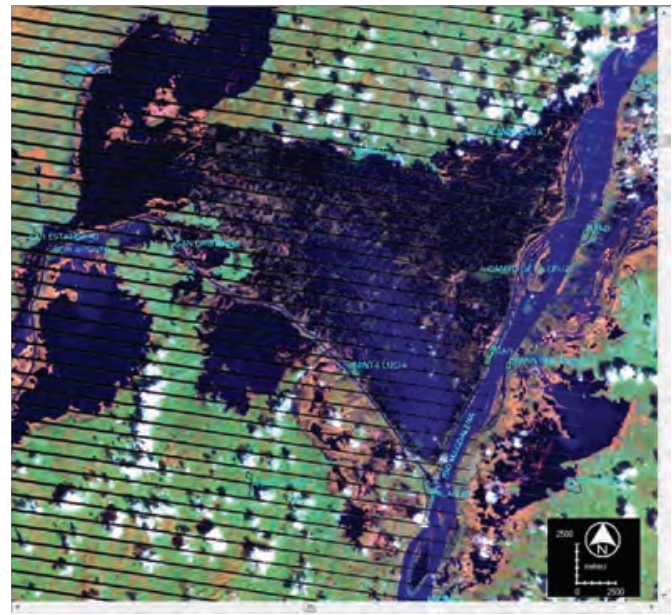

Figura 1c. Canal del Dique.

Figura 1a. Vista 3D de una imagen procesada Landsat ETM de 2009, mostrando el cráter y al fondo la ciudad de San Juan de Pasto. Earth Resources Observation and Science Center (EROS). USGS Global Visualization Viewer.

Figura 1b. Imágenes Landsat ETM y LDCM de enero de 2010. mostrando la zona antes de la inundación. Earth Resources Observation and Science Center (EROS). USGS Global Visualization Viewer.

Figura 1c. Imágenes Landsat ETM y LDCM de marzo de 2014, mostrando la zona inundada después de 36 meses de la inundación. Earth Resources Observation and Science Center (EROS). USGS Global Visualization Viewer.

de los mismos fenómenos de la zona alta, pero por ocurrencia de erupciones mayores, con probabilidad entre el 10 y $20 \%$ y que ocurran eventos entre 3 y 5 ; la tercera zona abarca la mayor área de los peligros volcánicos y, aunque afectaría con menor rigor a las comunidades, se encuentran áreas que se podrían afectar con una probabilidad de menos del $10 \%$, con una severidad igual o mayor a 2. La severidad está relacionada con la explosividad y depende del volumen de los productos expulsados y la altura de la nube eruptiva, que oscila entre 0 , con erupciones no explosivas y 8 , erupciones muy explosivas o terribles (Angeles et al. 2004; Linares et al. 1999).
En las zonas de amenaza alta y media, se encuentran campesinos e indígenas Quillasinga. López (2000) evidencia que en la zona existen problemas de limitado acceso a tierras para vivir y cultivar, tema a tener en cuenta en la gestión de riesgo planteada para la zona.

Amenazas y riesgos por inundaciones asociadas a la ruptura del Canal del Dique: En el 2010, se presentó el fenómeno de variabilidad climática "La Niña", con aumento significativo del caudal del río Magdalena. En noviembre del mismo año, este caudal hizo que la presión fallara sobre la estructura, generando una rotura en el jarillón. De acuerdo 
con Sánchez (2011), generó el ingreso de 2.200 millones de $\mathrm{m}^{3}$ de agua de las tierras bajas, ubicadas en el sur del departamento, con el resultado de la inundación del 10,4\% del departamento.

Esta rotura produjo la inundación de los municipios de Santa Lucía, Campo de La Cruz, Manatí, Suán y Candelaria y varios caseríos. Esta zona anegada, se presenta morfológicamente por debajo del Canal del Dique, en una antigua zona de ciénagas, que fueron desecadas.

La gobernación del Atlántico (2011) reportó 174.739 personas afectadas por la temporada invernal y las pérdidas económicas superaron, solamente en cultivos, los 400 mil millones de pesos (Figuras 1b y 1c).

Amenazas y riesgos por deslizamientos en Ciudad Bolívar - Bogotá: Para la capital, el área de montaña que corresponde a la zona de los cerros orientales, se caracteriza por una litología de rocas de composición arcillosa y arenosa, afectadas por fallas y pliegues; en conjunto con las fuertes pendientes de $20^{\circ}$ a $40^{\circ}$, las condiciones de drenajes inadecuados que saturan las laderas, hacen de éstas, áreas altamente susceptibles a desarrollar movimientos en masa, particularmente, para los barrios Perdomo Alto y Diamante, ubicados en Ciudad Bolívar, en donde una parte del cerro fue intervenida por minería de extracción de materiales de construcción; luego, fue objeto de rellenos, posteriormente, vino la urbanización masiva y, en los últimos diez años, afectado por deslizamientos.

\section{RESULTADOS Y DISCUSIÓN}

En este artículo, se abordó la relación entre imaginarios y percepciones sobre fenómenos naturales y la gestión del riesgo; para ello, se utilizaron categorías emergentes de marcos teóricos sobre la construcción socio-cultural del riesgo, aplicándolo a diferentes grupos poblacionales, como son habitantes urbanos, campesinos e indígenas, relacionados con diferentes fenómenos naturales.

Los resultados, se presentan por tipo de fenómeno analizado; la discusión posterior establece las relaciones posibles para la gestión del riesgo. Una síntesis de lo encontrado, se presenta en el cuadro 3, con los resultados por tipo de fenómeno estudiado, los actores directamente involucrados, sus percepciones e imaginarios sobre los fenómenos existentes en su zona de influencia y se analiza el aporte de estos imaginarios, para mejorar las estrategias de gestión del riesgo.

Imaginarios y percepciones con relación al sismo en Bogotá. La información de las campañas institucionales de prevención fue asumida diferencialmente por parte de los distintos pobladores de la ciudad, como se evidenció en las respuestas a las entrevistas realizadas, en el simulacro de octubre 2010. En general, existe una percepción de temor sobre la ocurrencia del sismo e incertidumbre sobre las acciones institucionales post-evento.

Imaginarios y percepciones sobre los fenómenos volcánicos. Los imaginarios y las percepciones de los indígenas Quillasingas y los campesinos respecto al volcán Galeras son de cercanía hacia el fenómeno; para los primeros, el volcán es un padre benefactor, que lo llaman Urcunina y, para los segundos, es un fenómeno que les ha enriquecido sus suelos, facilitando los cultivos y la provisión de alimentos en las tierras abonadas, con ceniza volcánica.

En cuanto al volcán Nevado del Huila, la avalancha ocurrida en 1994, fue para los indígenas Nasa un aviso o advertencia que les llamó la atención acerca de la desorganización como comunidad; para el $50 \%$ de los miembros de la comunidad entrevistados, la desorganización de la comunidad no permitió que naciera otro héroe cultural, como fue Juan Tama, planteamiento enunciado también por Wilches-Chaux (2005). El 50\% de los representantes de la comunidad Nasa entrevistados, el Nevado es un preceptor, que puede castigarlos por su falta de organización y la pérdida de su conocimiento tradicional, por ello, deben estar atentos, a través de bio-indicadores acerca de cualquier cambio que ocurra en el fenómeno.

Respuestas locales ante el fenómeno de inundación. Las personas afectadas por inundaciones en los municipios del Canal del Dique han perdido la memoria sobre otros eventos similares en el pasado, por ejemplo, el ocurrido en 1984. Una de las razones aducidas para ocupar estas zonas de inundación es la necesidad de estar cerca de sus familiares, lo cual, les da una relativa seguridad económica, por los lazos de solidaridad establecidos.

Para los campesinos de Tinjacá en Boyacá, el río es una amenaza y con esa concepción, se tiene el imaginario que talando los árboles en la ribera se pueden evitar las inundaciones; también el $60 \%$ de los campesinos entrevistados, en este municipio, piensa que una solución es dragar el río, sacar el material depositado allí y comercializarlo.

Imaginarios y percepciones sobre movimientos en masa, Localidades de Rafael Uribe y Ciudad Bolívar, Bogotá. Los fenómenos de remoción en masa estudiados, se caracterizan por ser lentos, por tanto, las comunidades han generado un acostumbramiento frente al fenómeno. Muchas veces, se minimiza el riesgo frente a otras necesidades de orden económico, reflejadas en la búsqueda de un ingreso diario, para su sustento. Para el $30 \%$ de las personas participantes de los talleres en Ciudad Bolívar, las causas del fenómeno son las inadecuadas intervenciones que se han realizado sobre 
el territorio por parte de empresas, como la de Acueducto y Alcantarillado. También, se critica el control que se hace sobre el territorio con relación a la ocupación de zonas de riesgo. En la localidad Rafael Uribe, a pesar de la asistencia que hacen los funcionarios del Fondo de Prevención y Atención de Emergencias -FOPAE- y la Caja de Vivienda Popular, las personas son escépticas frente a la acción institucional.

\section{Discusión de resultados hacia el enriquecimiento de la gestión del riesgo}

Posiciones de los seres humanos frente a la naturaleza. Los grupos indígenas estudiados evidencian un acercamiento a una concepción de naturaleza holística; los campesinos de la zona del Galeras tienen puntos de acercamiento con esta concepción. Retomando los planteamientos de Mebratu (2001) y Capra (1998), la concepción holística rompe con el pensamiento racional dualista de separación, entre el ser humano y la naturaleza, de los expertos en fenómenos naturales y de algunos de los funcionarios acreditados para su gestión.

Los campesinos entrevistados de Boyacá y los habitantes urbanos tienen una visión más cercana a una posición de separación con la naturaleza, que transita hacia una concepción sobre naturaleza, como recurso a explotar, encontrada en el caso del río, como fuente de material a comerciar y en la explotación de canteras en el medio urbano, generando escenarios de riesgo.

Un aspecto fundamental en los programas y proyectos de gestión del riesgo son las razones que tienen las personas afectadas para quedarse en la zona de amenaza, relacionados con la posibilidad de perder su tejido social, la inseguridad y el miedo a la discriminación, que creen podrán sufrir al llegar a otro sitio. Esto se plantea con relación a los campesinos del volcán Galeras frente a los representantes de la Unidad de Gestión del Riesgo, quienes los invitaron a participar y conocer sus necesidades.

En comunidades urbanas, particularmente, es evidente la existencia de factores económicos que han impedido acceder a suelos urbanos adecuados para los asentamientos. Aunado a ello, se generan problemas de justicia ambiental y de percepción del riesgo, que se convierte en problemas de justicia espacial, en términos de lo planteado por Musset (2009). En efecto, según el autor, existen procesos geohistóricos de intervención del territorio, donde se van configurando escenarios de riesgo, debido a decisiones de política económica y de ordenamiento territorial, que generan situaciones de desigualdad. Así, además de enfrentarse a riesgo de movimiento en masa, los pobladores de estos lugares se deben enfrentar a los impactos de proyectos urbanos, como por ejemplo, un relleno sanitario, un cementerio como lo re- latado por las personas afectadas, que va en consonancia con lo planteado por Kuclicke (2007), acerca de la existencia de lo que se ha denominado como "puntos calientes", es decir, territorios donde confluyen fenómenos naturales amenazantes y situaciones de pobreza y violencia, lo que aumenta las condiciones de vulnerabilidad.

Conocimiento local. Al retomar los planteamientos de Watts (2003), sobre un conocimiento "cosmopolita", se evidencia, en el caso de los indígenas, la existencia de una memoria histórica remontada a épocas de preconquista; también se refleja en ese conocimiento fenomenológico sobre el territorio de los grupos Nasa y el hallazgo de puntos de convergencia con el conocimiento experto, para monitorear y prevenir situaciones desastrosas.

Respecto a las comunidades urbanas, los resultados permiten evidenciar que los procesos de migración intra-urbana y el desplazamiento, han propiciado que, como lo señala Kuclicke (2007), se pierda esa memoria sobre lo que ha acontecido en el territorio. Muchas veces, las personas desconocen que hace unos años esas zonas eran explotaciones de canteras.

Imaginarios y concepciones sobre la gestión del riesgo. Dentro de las críticas presentadas por las personas entrevistadas, se evidencia lo planteado por Lampis (2012), acerca de la existencia de diversos intereses de los diferentes actores sociales (Cuadro 2), asociados a la gestión del riesgo y que ha denominado como "dobles agendas", lo cual, se acerca a los desencuentros entre imaginarios e instituciones, según lo planteado por Maskrey (1994). Esto se puede corroborar, con lo acontecido en el caso del volcán Galeras, pues los campesinos sienten que la reubicación es una amenaza para ellos, por la pérdida de su tejido social. En el caso de Bogotá, se evidencia en la percepción que tienen algunos habitantes urbanos acerca de que los deslizamientos fueron ocasionados por la intervención del acueducto o que los proyectos de la Caja de Vivienda Familiar no responden a sus urgencias de solución.

Se resalta que si bien existen problemas de comunicación y desencuentros nacidos de diferentes cosmovisiones, también es cierto que existen problemas estructurales, como lo plantea Blakie et al. (1996), Cannon (2008), Musset (2009), relacionados con temas de desarrollo-y de desigualdad, que se agrava con injusticia espacial, que deben ser tenidos en cuenta al momento de revisar las gestiones que se realicen en los ámbitos locales.

Las racionalidades planteadas por Wildavsky (1998). Los resultados (Cuadro 3) muestran que los indígenas tienden a ser más igualitarios, lo mismo que algunos grupos de campesinos, debido a su tradición histórica, su relación con la 
Cuadro 2. Actores sociales y características.

\begin{tabular}{|c|c|c|}
\hline $\begin{array}{l}\text { Actores } \\
\text { sociales }\end{array}$ & Características & $\begin{array}{l}\text { Número de } \\
\text { entrevistas y } \\
\text { distribución de } \\
\text { porcentajes }\end{array}$ \\
\hline $\begin{array}{l}\text { Campesinos } \\
\text { Zona Canal } \\
\text { del Dique } \\
\text { (Atlántico) }\end{array}$ & $\begin{array}{l}\text { Se dedican al sector agropecuario cultivan, yuca, maíz, frijol, ahuyama y frutales, } \\
\text { como melón y patilla y cítricos. Complementan con la cría de especies menores, } \\
\text { la elaboración y venta de alimentos en el espacio público. Los hombres se encar- } \\
\text { gan de la producción y las mujeres del cuidado del hogar, de los niños y de la cría } \\
\text { de las especies menores (Hurtado, 2011). Para la subregión de la que hacen parte } \\
\text { los municipios afectados por las inundaciones del Canal del Dique, se encuentra } \\
\text { que } 104.161 \text { personas es población perteneciente al SISBEN (Sistema de Identi- } \\
\text { ficación y Clasificación de Potenciales Beneficiarios para los programas sociales), } \\
\text { o sea, personas que no pueden cubrir sus necesidades básicas y el NBI (Índice } \\
\text { de Necesidades Básicas Insatisfechas) es del } 59,01 \%, 62,17 \% \text {, en la cabecera y } \\
52,07 \% \text {, resto (Hurtado, 2011). }\end{array}$ & \multirow{7}{*}{$\begin{array}{l}\text { En total se realizaron } \\
107 \text { entrevistas } \\
\text { discriminadas así: } \\
\\
\text { Por sexo: } \\
45 \% \text { de hombres y } \\
55 \% \text { de mujeres; } \\
\\
\text { Por edad: } \\
4 \% \text { de niños, } 5 \% \\
\text { adolescentes, } 84 \% \\
\text { de adultos y } 7 \% \text { de } \\
\text { adultos mayores; } \\
\\
\text { Por dedicación: } \\
23 \% \text { dedicadas a } \\
\text { labores del hogar, } \\
5 \% \text { líderes urbanos, } \\
46 \% \text { personas } \\
\text { con diferentes } \\
\text { ocupaciones, } \\
5 \% \text { funcionarios, } \\
10 \% \text { indígenas y } 11 \% \\
\text { de campesinos. }\end{array}$} \\
\hline $\begin{array}{l}\text { Municipio } \\
\text { de Tinjacá } \\
\text { (Boyacá) }\end{array}$ & $\begin{array}{l}\text { Tienen una importante tradición muisca. Cultivan el maíz, la papa, la yuca. Prac- } \\
\text { tican la agricultura de subsistencia, principalmente del maíz; en los últimos años } \\
\text { empezaron a cultivar el tomate. Complementan sus actividades con el cuidado de } \\
\text { ganado bovino, ovino y algunos frutales. }\end{array}$ & \\
\hline $\begin{array}{l}\text { Genoy } \\
\text { (Nariño) }\end{array}$ & $\begin{array}{l}\text { La población total de este corregimiento es de } 1.244 \text { habitantes. Se dedican a } \\
\text { la agricultura de trigo, maíz, ulloco, arveja, cebada, hortalizas. Proyecto Código } \\
\text { Periferia Urbana ARD (2003). }\end{array}$ & \\
\hline $\begin{array}{l}\text { Indígenas } \\
\text { Quillasingas } \\
\text { (Nariño) }\end{array}$ & $\begin{array}{l}\text { Se ubican en los alrededores de Pasto, principalmente, en el corregimiento de } \\
\text { Genoy. Utilizan diferentes pisos términos para su seguridad agroalimentaria. } \\
\text { Tienen pequeñas fincas en las zonas calidad o de sierra, para la producción de } \\
\text { maíz, caña de azúcar, café, cabuya, frutales, plátano y yuca (López, 2000). }\end{array}$ & \\
\hline Nasa (Cauca) & $\begin{array}{l}\text { Se ubican en el municipio de Paéz (Cauca). Sus formas de producción se basan } \\
\text { en un calendario natural que marca las épocas de cosecha, con } 20 \text { épocas natu- } \\
\text { rales, relacionadas con el cultivo del maíz y cuatro actividades rituales (Osorio, } \\
\text { 2009). }\end{array}$ & \\
\hline $\begin{array}{l}\text { Ciudad Bolívar } \\
\text { (Bogotá) }\end{array}$ & $\begin{array}{l}\text { La localidad presenta un modelo de desarrollo de carácter informal con el mayor } \\
\text { número de necesidades básicas insatisfechas en Bogotá. Es la primera localidad } \\
\text { en recibir personas desplazadas del país, lo cual, incide en las altas tasas de po- } \\
\text { breza y de miseria. El estrato bajo - bajo es el más representativo de la localidad } \\
\text { con una representación del 58,41\% (Alcaldía Mayor de Bogotá, 2009). }\end{array}$ & \\
\hline $\begin{array}{l}\text { Rafael Uribe } \\
\text { (Bogotá) }\end{array}$ & $\begin{array}{l}\text { La localidad tiene } 377.615 \text { habitantes. La pirámide poblacional presenta una } \\
\text { base reducida con menor proporción de niños, con mayor número de población } \\
\text { en edades intermedias (Hospital Rafael Uribe Uribe, 2009). La localidad, se pre- } \\
\text { senta como la quinta con menor nivel de vida de la ciudad, pues a pesar de con- } \\
\text { tar con una buena cobertura en los servicios públicos, en términos de cantidad } \\
\text { y calidad, la zona se caracteriza por presentar hacinamiento de los hogares. El } \\
\text { tamaño promedio del hogar en la localidad es de } 3,63 \text { personas, mayor al de la } \\
\text { ciudad, que en promedio es de } 3,4 \text { personas por hogar, (Secretaría Distrital de } \\
\text { Planeación, 2009). }\end{array}$ & \\
\hline
\end{tabular}


Cuadro 3. Síntesis de resultados.

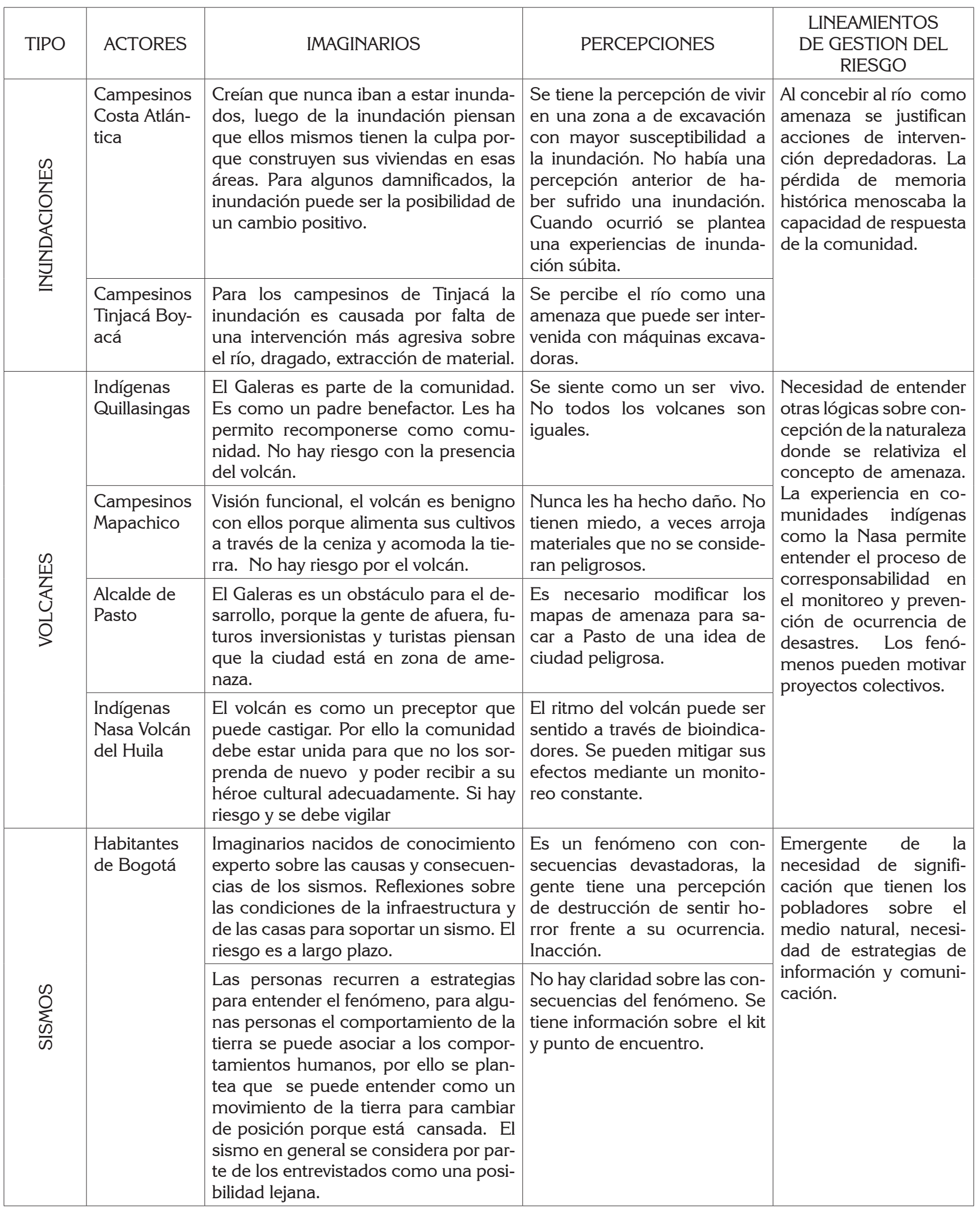


Continuación cuadro 3

\begin{tabular}{|c|c|c|c|c|}
\hline 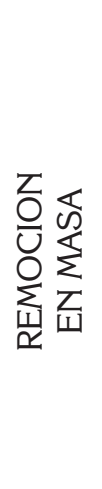 & $\begin{array}{l}\text { Habitantes } \\
\text { Ciudad Bolí- } \\
\text { var. Bogotá }\end{array}$ & $\begin{array}{l}\text { Los movimientos ocurren por la inter- } \\
\text { vención inadecuada de entidades, para } \\
\text { los habitantes de las localidades estu- } \\
\text { diadas, muchos de los deslizamientos } \\
\text { son generados por la intervención del } \\
\text { terreno por parte de entidades como } \\
\text { la Empresa de Acueducto y Alcantari- } \\
\text { llado. Otros plantean que ocurre por } \\
\text { falta de planificación y control oficial } \\
\text { sobre el manejo del suelo. Miedo a } \\
\text { perder lo que se tiene más que el mie- } \\
\text { do a perder la vida. }\end{array}$ & $\begin{array}{l}\text { Desconfianza ante la gestión } \\
\text { del Estado. Se percibe como } \\
\text { un fenómeno cotidiano con el } \\
\text { cual convivir. Hay otras prio- } \\
\text { ridades cotidianas, existe un } \\
\text { miedo a perder lo poco que } \\
\text { se ha conseguido. Inseguri- } \\
\text { dad del porvenir. Miedo rela- } \\
\text { tivo }\end{array}$ & $\begin{array}{l}\text { Intervenciones negati- } \\
\text { vas sobre el medio na- } \\
\text { tural, inacción para ha- } \\
\text { bitantes. Importancia } \\
\text { de la norma para con- } \\
\text { trolar y gestionar. Pérdi- } \\
\text { da de memoria sobre lo } \\
\text { ocurrido en el territorio. }\end{array}$ \\
\hline
\end{tabular}

tierra y su sentido de grupo. Por su parte, los funcionarios tienden a tener una racionalidad jerárquica, con su sentido de la norma, el protocolo y la necesidad del control. Asimismo, algunos personas con prevalencia de racionalidad individualista, según lo manifestado por los entrevistados del Atlántico, buscaron solamente su beneficio personal y no fueron solidarios con los otros; por otro lado, en la zonas de afectación por fenómenos de remoción en masa e inundaciones, se encuentran racionalidades más de tipo fatalista y los afectados se sienten inermes frente al Estado, con bajos niveles de conformación de redes sociales sólidas.

El vínculo entre estas racionalidades y la política pública sobre gestión del riesgo es clave y concuerda con lo planteado por Losada (2010). No es posible estructurar una política pública que responda a una sola racionalidad. Por tanto, se puede trabajar en un marco lo suficientemente amplio, que permita tomar lo mejor de estas racionalidades y estructurar una estrategia que facilite su incorporación. A decir de Schwarz \& Thompson (1990) y de Wildavsky (1998) todas existen como una especie de mosaico y son necesarias en una política integral de gestión del riesgo.

Conflictos de intereses: El manuscrito fue preparado y revisado con la participación de los autores, quienes declaramos que no existe conflicto de intereses, que ponga en riesgo la validez de los resultados presentados. Financiación: Este estudio fue financiado por la Universidad Nacional de Colombia, a través del grupo de Investigación Geotecnologías.

\section{BIBLIOGRAFÍA}

1. ALCALDÍA MAYOR DE BOGOTÁ. 2009. Localidad Ciudad Bolívar. Diagnóstico local con participación social 2009-2010. Bogotá. 335p.

2. ANGELES, l.; ORTIZ, R.; MARRERO, J.M. 2004. Riesgo Volcánico. Dirección Nacional de Protección civil y emergencias. Imprenta Nacional del Boletín oficial del Estado. Madrid.103p.

3. BERGER, P.; LUCKMANN, T. 2001. La Construcción social de la realidad. Amorrortu editores. Buenos Aires. 240p.

4. BLAKIE, P.; CANNON, T.; DAVIS, E.; WISNER, B. 1996. Vulnerabilidad. El entorno social, político y económico de los desastres. Red de Estudios Sociales en Prevención de Desastres en América Latina. 292p.

5. CANNON, T. 2008. Reducing People's Vulnerability. Communities and Resilience-.London School of Humanities and Natural Resources Institute, University of Greenwich. United Nations University. London. $19 p$.

6. CANNON, T.; MÜLLER-MAHN, D. 2010. Vulnerability, resilience and development discourses in contexto of climate change. International Institute for Environment and Development. London. 15p.

7. CAPEL, H. 1973. Percepción del medio y comportamiento geográfico. Rev. Geografica. España. 7(1):58-150.

8. CAPRA, F. 1998. El Punto Crucial.: Editorial Estaciones. Buenos Aires. 247p.

9. DOUGLAS, M. 1996. La aceptabilidad del riesgo según las ciencias sociales. Ed. Paidos Ibérica. Barcelona. $176 p$.

10. ESCOBAR, A. 2011. Epistemologías de la naturaleza y la colonialidad de la naturaleza. Variedades de realismo y constructivismo. En: Cultura y Naturaleza. Ed. Montenegro, L. Jardín Botánico de Bogotá José Celestino Mutis. (Bogotá). 472p. 
11. GARCÍA, V. 2005. El riesgo como construcción social y la construcción social de riesgos. Desacatos. Rev. Antopol. Social. México. 19:11-24.

12. GOBERNACIÓN DEL ATLÁNTICO. 2011. Población damnificada por inundaciones 2010-2011. Disponible desde internet en: http://www.atlantico. gov.co/index.php?option $=$ com_content $\&$ view $=$ ar ticle\&id=722: poblacion\&catid = 125: informaciongeneral\&Itemid=491 (con acceso13/01/2011).

13. HAMMERSLEY, M.; ATKINSON, H. 1994. Etnografía. Métodos de investigación. Ed.Paídos. (Barcelona) 352p.

14. HOSPITAL RAFAEL URIBE URIBE. 2009. Indicadores de diagnóstico ASIS. Disponible desde internet en: http://www.eserafaeluribe.gov.co/asis/diagnosticos. php (con acceso 23/10/2009).

15. HURTADO, I. 2011. Evaluación de inundaciones departamento del Atlántico.2010. Instituto de estudios humanitarios. Colombia. 38p.

16. KUCLICKE, C. 2007. (Non-)Knowledge in Hazard and Vulnerability Research. En: Warner, K. (ed). Perspectives on Social Vulnerability. Bonn: UNU-EHS. 131p.

17. LAMPIS, A. 2012. La adaptación al cambio climático. El reto de las dobles agendas. En: Postigo, J. Cambio Climático, movimientos sociales y políticas públicas una vinculación necesaria. Instituto de Ciencias Alejandor Lipschutz (ICAL). Chile. p.27-47.

18. LINARES, E.; OSTERA, H.A.; MAS, L.C. 1999. Cronología K-Ar del Complejo Efusivo Copahue- Caviahue, Provincia del Neuquén. Rev. Asoc. Geológica Argentina. 54:240-247.

19. LINDON, A. 2007. Los imaginarios urbanos y el constructivismo geográfico: Los hologramas espaciales. Rev. Eure (Santiago de Chile). 33(99):31-46.

20. LÓPEZ, C. 2000. Pueblos del Valle de Atrís. En Geografía humana de Colombia, Región Andina Central. Tomo IV, volumen I. Bogotá: Edición original. Instituto Colombiano de Cultura Hispánica. Disponible desde Internet en: http://www.banrepcultural.org/blaavirtual/geografia/geohum4/atris1.htm (con acceso 31/02/2010).

21. LOSADA, R. 2010. La percepción y el análisis de riesgos. En: Ignacio, C.J. (ed). Castástrofes medioambienta- les. La reacción social y política. Tirant Lo Blanch. Valencia. p.75-150.

22. MASKREY, A. 1994. Comunidad y Desastres en América Latina. En: Allan, L. (ed). Viendo en Riesgo. FlacsoLa Red-Tercer Mundo. Bogotá. p.14-38.

23. MEBRATU, D. 2001. The knowledge dimension of the sustainability chalenge. International institute for Industrial Environ. Econom. Lund University. Suecia. 21p.

24. MORA, J. 2008. Persistencia, conocimiento local y estrategias de vida en sociedades campesinas. Rev. Estudios Sociales. 29:122-133.

25. MUSSET, A. 2009. ¿'Geohistoria o geoficción?. Ciudades vulnerables y justicia espacial. Universidad de Antioquia. Medellín. 266p.

26. NAJMANOVICH, D. 2008. Mirar con nuevos ojos. Biblos. Buenos Aires. 200p.

27. OSORIO, G. 2009. Cosmovisión de pueblo indígena Nasa en Colombia. Reducción integral de los riesgos, planificación y desarrollo sostenible. Predecan. Proyecto Prevención de desastres en la comunidad andina. (Lima). 36p.

28. Proyecto Código Periferia Urbana. ARD-UDENAR. 2003. Pasto. Nariño. Disponible desde internet en: http:// www.revistaescala.com/attachments/197_7.\%20 Diagnostico\%20Centros\%20Poblados\%20ämr.pdf (con acceso el 01/03/2012).

29. RABEY, M. 1990. Conocimiento popular y desarrollo. Medio ambiente y Urbanización. p.46-55.

30. SÁNCHEZ, A. 2011. Después de la Inundación. Documentos de trabajo sobre economía regional. Banco de la República.Centro de Estudios Económicos Regionales. Cartagena. 50p.

31. SCHIFFMAN, H. 2004. Sensación y percepción. Un enfoque integrador. Ed. Manual Moderno. México. 562p.

32. SERVICIO GEOLÓGICO COLOMBIANO. OBSERVATORIO PASTO. 2011. Mapa de amenaza volcánica del Galeras Tercera versión memoria explicatival. Disponible desde internet en: http://www.sgc.gov.co/Pasto/ Volcanes/Volcan-Galeras/Mapa-de-amenazas.aspx. (con acceso 02/05/2014). 
33. SECRETARÍA DISTRITAL DE PLANEACIÓN. 2009. Conociendo la localidad de Rafael Uribe Uribe, diagnóstico de los aspectos físicos, demográficos y socioeconómicos. Bogotá: Alcaldía Mayor de Bogotá. $16 \mathrm{p}$.

34. SCHWARZ, M.; THOMPSON, M. 1990. Divided we Stand. Redifining politics, technology and social choice. University of Pennsylvania Press. Pennsylvania. 176p.

35. TUAN, Y.F. 2007. Topofilia. Melusina. España.351p.

36. WATTS, M. 2003 Alternative Modern-Development as cultural Geography. En K. Anderson, M. Domosh, \& P. Steve, Handbook of Cultural Geography. London: Sage. p.433-453.
37. WILCHES-CHAUX, G. 2005. Proyecto Nasa: Las construcción del plan de vida de un pueblo que sueña. Programa de Naciones Unidas para el Desarrollo. Bogotá. 159p.

38. WILDAVSKY, A. 1998. Culture and social theory. Transaction Publisher. (New Jersey). 546p

39. WISNER, B.; BLAIKIE, P.; CANNON, T.; DAVIS, I. 2003. At Risk: Natural Hazards, peoples's Vulnerability and disasters. Routledge. London y New York. 447p.

Recibido: Abril 21 de 2014

Aceptado: Octubre 9 de 2014

\section{Como citar:}

Hernández Peña, Y.T.; Vargas Cuervo, G. 2014. Análisis de respuestas locales ante fenómenos naturales amenazantes y dinámicas de construcción de escenarios de riesgo en Colombia. Rev. U.D.C.A Act. \& Div. Cient. 17(2): 529-539. 